ANALES CERVANTINOS, VOL. XLVIII,

PP. 361-367, 2016, ISSN: 0569-9878, e-ISSN:1988-8325

doi: 10.3989/anacervantinos.2016.014

\title{
Cervantes y el CSIC
}

\section{María del Pilar Martínez Olmo*}

\begin{abstract}
Resumen:
El Consejo Superior de Investigaciones Científicas conmemora el IV centenario de la muerte de Miguel de Cervantes organizando varias actividades científicas y divulgativas que tendrán lugar en la sede de Madrid y en las delegaciones de diferentes comunidades autónomas. En esta nota se describen las más destacadas y especialmente la exposición Cervantes y el CSIC, celebrada en Madrid durante el mes de abril de 2016.
\end{abstract}

Palabras clave: Cervantes; IV Centenario; CSIC; Exposiciones.

Title: Cervantes and the Spanish National Research Council

\begin{abstract}
:
The Spanish National Research Council commemorates the Forth Centenary of Miguel de Cervantes' Death organizing academic events and exhibitions in Madrid and in different provinces. This note describes the most relevant activities and, in particular, the exhibition Cervantes y el CSIC, celebrated in Madrid, April 2016.
\end{abstract}

Key Words: Cervantes; IV Centenary; CSIC; Expositions.

El Consejo Superior de Investigaciones Científicas (CSIC) ha promovido desde su fundación las investigaciones sobre Miguel de Cervantes y ha contribuido a un mejor conocimiento de su vida y de su obra. Es además editor de la única revista española dedicada al genio de nuestras letras, Anales Cervantinos, y en su Red de Bibliotecas y Archivos se reúnen algunos ejemplares únicos y otros raros y valiosos que permiten conocer mejor la difusión de la obra del Príncipe de los Ingenios.

*Biblioteca Tomás Navarro Tomás, CCHS, CSIC. pilar.martinez@cchs.csic.es 
Durante 2016, coincidiendo con la celebración del IV centenario de su muerte, el CSIC ha programado varias actividades que destacan la presencia de Cervantes en la Ciencia, destacar las publicaciones realizadas por el personal investigador de la institución que ha profundizado en la persona, el escritor y el mito. Asimismo, dar a conocer la colección cervantina reunida desde los años cuarenta del siglo XX, y que cuenta con ediciones y estudios desde 1631 hasta nuestros días.

Cervantes y el CSIC es el título que unifica las diferentes actividades. Una exposición organizada en la sede central del organismo en Madrid, una exposición virtual y un audiovisual accesibles a través de Internet, la edición de Viaje del Parnaso y Una ingeniosa locura. Libros y erudición en Cervantes, ciclos de conferencias, exposiciones en diferentes delegaciones del CSIC en comunidades autónomas, un recorrido por las plantas que aparecen en las obras de Cervantes o la lectura dramatizada de Palabra de perro, obra del dramaturgo Juan Mayorga, inspirada en El coloquio de los perros de Cervantes, son algunas de las actividades más destacadas que ha organizado el CSIC en este cuarto centenario.

La exposición Cervantes y el CSIC, abierta al público durante el mes de abril de 2016 en la sede central del organismo en la calle Serrano 117 de Madrid, ha sido coordinada por la Biblioteca Tomás Navarro Tomás y en ella han participado, cediendo ejemplares de sus fondos, las bibliotecas de la Institución Milá y Fontanals (Barcelona), Escuela de Estudios Árabes (Granada), Escuela de Estudios Hispanoamericanos (Sevilla), Real Jardín Botánico e Instituto Cajal (Madrid), así como la Unidad de Recursos de Información Científica para la Investigación (URICI). Una combinación de paneles explicativos, ejemplares bibliográficos y documentos de archivo ha permitido ofrecer una visión amplia de la presencia de Cervantes en diferentes Ciencias y dar a conocer la colección cervantina reunida en el $\mathrm{CSIC}^{1}$.

$\mathrm{Su}$ estructura gira en torno a tres ejes precedidos de una breve introducción y un apartado que recoge los escasos datos que conocemos de la biografía de Cervantes y en especial sobre su estancia como cautivo en Argel. Ejemplares de la Historia del reyno de Argel, de Laugier de Tassy, o la Topografía de historia de Argel de Diego de Haedo, han servido para ilustrar el panel explicativo titulado Cervantes, don Quijote y el Mediterráneo ${ }^{2}$.

La primera sección se centra en la importancia de Cervantes como modelo e inspiración en diferentes disciplinas científicas. Sus obras son reflejo de una época rica en ciencia y cultura. En ellas se encuentran referencias a la naturaleza, al paisaje, la alimentación, la medicina, las ciencias, la vida de los

1. Los paneles explicativos han sido redactados por especialistas en cada materia. En la exposición participan científicos como José Luis Peset, Luciano García Lorenzo, Miguel Ángel de Bunes, Mariano Lambea, Joaquín Álvarez Barrientos y Alfredo Alvar. Los técnicos de la BTNT han redactado los textos relativos a la colección bibliográfica y documental. Ana Jiménez firma el texto de Anales Cervantinos, Gloria Lence la sección de bibliografía y Rosa María Villalón y Raquel Ibáñez han redactado la información sobre el archivo de Francisco Rodríguez Marín.

2. Laugier de Tassy [1750] y De Haedo (1612). 
cautivos, el entorno rural y la vida de la corte, todo ello transmitido con un lenguaje rico en registros. Y, gracias a la calidad de sus escritos y a la difusión que alcanzaron sus obras, hoy la situación se ha dado la vuelta y encontramos referencias a Cervantes en medicina, música, arte, etc.

Junto a la obra de Juan Valverde de Hamusco y una edición de la Historia general y natural de las Indias de Fernández de Oviedo, ejemplos ambos de los avances científicos de la época de Cervantes, la exposición cuenta con ediciones de Los primores de don Quijote, de Pi y Molist, el Tratado completo de las enagenaciones mentales de Esquirol o el Examen de ingenios para las ciencias de Juan Huarte. Destaca el ejemplar del discurso pronunciado en 1905 por Santiago Ramón y Cajal sobre Psicología de Don Quijote y el quijotismo, junto con algunos estudios recientes del profesor Peset que proyectan la presencia de Cervantes hasta el siglo $\mathrm{XXI}^{3}$.

La música también ha tenido en Cervantes una fuente de inspiración y compositores de varias generaciones, no solo españoles sino de diferentes nacionalidades y estilos, han compuesto música sinfónica, ópera, ballet o música de cámara inspirados muy especialmente, aunque no de forma única, en el héroe de La Mancha. Una selección de obras del fondo de la Institución Milá y Fontanals en diferentes soportes acompaña la explicación proporcionada en el panel por el doctor Mariano Lambea. El público asistente ha podido contemplar partituras, grabaciones y estudios de las composiciones de Falla, Albéniz, Purcell o Mendelssohn. Destaca por su belleza la partitura de Jan Fischer Lisâk Pedro Napsal ${ }^{4}$ y por su contenido el disco compacto Entre aventuras y encantamientos ${ }^{5}$, realizado por científicos de la Institución Milá y Fontanals (CSIC) con una selección de obras musicales de la época de don Quijote.

Dentro de la sección dedicada a la presencia de Cervantes en diferentes ciencias, se han querido destacar también algunos de los homenajes realizados dentro del CSIC como el Phrydiuchus quijote o el Nanoquijote. Los trabajos de un equipo de investigación del Museo Nacional de Ciencias Naturales permitieron identificar una especie nueva de coleóptero en La Mancha. Los entomólogos que descubrieron los ejemplares, Manuel Sánchez Ruiz y Miguel Ángel Alonso Zarazaga, le pusieron el nombre de Phrydiuchus quijote ${ }^{6}$ en homenaje a su personaje más célebre. En el Instituto de Ciencias de Materiales de Madrid encontramos el Nanoquijote ${ }^{7}$, creado por el doctor Ricardo García

3. Valverde de Hamusco (1556); Fernández de Oviedo (1851-1855); Pi y Molist (1886); Esquirol (1847); Huarte (1883); Ramón y Cajal (1905); Peset (2010 y 2015).

4. Fischer [1958].

5. Entre aventuras y encantamientos [Grabación sonora musical]: música para don Quijote, [Madrid], Lauda Música, D.L. 2005.

6. Puede consultarse el vídeo elaborado por la biblioteca Tomás Navarro Tomás en la dirección electrónica https://www.youtube.com/watch?v=QkBajaTQKdk [Consultado el 21-06-2016]

7. Puede consultarse el vídeo elaborado por la biblioteca Tomás Navarro Tomás en la dirección electrónica https://www.youtube.com/watch?v=UpQQNyujUWc [Consultado el 21-06-2016] 
García como homenaje a Cervantes en 2005. En la vitrina de la exposición se ha expuesto la placa de silicio en la que, con técnicas de nanotecnología, se realizó una impresión del primer párrafo del Quijote. Aplicando estas técnicas, el Quijote podría estar escrito en la punta de un cabello.

Pero, aunque en todas las ciencias encontramos referencias a Cervantes, es en la literatura de los siglos XVIII a XXI donde encontramos un número elevado de creaciones nacidas bajo la influencia de sus obras: Historia del más famoso escudero Sancho Panza, La gitanilla en Madrid, El nuevo coloquio de los perros o La quijotita y su prima, son algunas de ellas ${ }^{8}$. De la mano de Joaquín Álvarez Barrientos se reconstruye el itinerario que ha llevado a Cervantes a ser modelo no solo literario y moral sino también a encarnar los valores nacionales. Pero esta merecida fama motivó que fuera víctima de plagios y atribuciones erróneas. En el panel "Entre falsos anda el juego" Álvarez Barrientos explica los casos de Avellaneda o El buscapié, ${ }^{9}$ que pudieron verse también en las vitrinas de la exposición.

El segundo eje muestra la importancia que Cervantes tiene dentro del CSIC y las aportaciones que se han realizado para mejorar el conocimiento de su vida y su obra. El Instituto Miguel de Cervantes de Filología Hispánica, la revista Anales Cervantinos o el congreso realizado en 2005 sobre El Quijote y el pensamiento teórico-literario son algunos ejemplos. Pocos años después de la creación del CSIC, se tomó la decisión de crear un instituto dedicado a los estudios filológicos que llevara el nombre de Miguel de Cervantes. Documentos de trabajo, fotografías, cartas, manuscritos, pruebas de imprenta y sellos han permitido acercar al visitante una forma de trabajar no tan lejana pero diferente a la de nuestros días. Destaca entre sus publicaciones la revista Anales Cervantinos, única publicación dedicada íntegramente al autor del Quijote y, como muestra de su trayectoria, iniciada en 1951, la exposición contaba con una selección de números con las contribuciones de literatos como Gerardo Diego (primer artículo del primer número de 1951) o Antonio Muñoz Molina (volumen 37 de 2005), aportaciones de científicos como Luciano García Lorenzo, Alberto Sánchez, etc. La exposición cuenta también con cubiertas de números de diferentes épocas, ejemplos de una evolución estética de más de medio siglo hasta llegar a su versión electrónica actual, a la que se podía acceder a través de un código QR. En el portal digital se amplía la información de le exposición física al ofrecer una bibliografía de todos los artículos publicados en Anales desde 1951, realizada en la biblioteca Tomás Navarro Tomás por Ana Jiménez Royo ${ }^{10}$.

El tercer eje de la exposición Cervantes y el CSIC se dedica a la colección cervantina del CSIC. Está formada por ejemplares bibliográficos y un fondo

8. Gatell (1794); Solís (1780); Benavente (1908); Fernández de Lizardi (1942).

9. Obra de Adolfo de Castro, se publicó como inédito de Cervantes. Se ha expuesto un ejemplar de la edición de Cádiz, Imprenta de Rev. Médica, 1848.

10. Puede consultarse en http://biblioteca.cchs.csic.es/dia_libro_cervantes_2016/anales.html [Consultado el 21-06-2016] 
archivístico de especial relevancia: el Archivo Rodríguez Marín. Se han seleccionado más de una veintena de ediciones de Cervantes entre las más de 800 existentes en la Red de Bibliotecas y Archivos del CSIC. La más antigua conservada es una edición de las Novelas ejemplares de 1631 pero quizás la más destacada sea el ejemplar del Quijote de 1738, realizada en Londres por J. y R. Tonson, y que va acompañada de más de sesenta grabados a página completa, acorde con las ideas estéticas de la Inglaterra del siglo XVIII ${ }^{11}$. Además, se han expuesto ediciones académicas, ilustradas, adaptaciones para niños y una selección de traducciones a otras lenguas algunas de las cuales utilizan también alfabetos no latinos (árabe, hebreo, etc.). Del Archivo Rodríguez Marín se han seleccionado documentos, fotografías, recordatorios de celebraciones en honor de Cervantes y varios documentos relativos a la selección del proyecto para el monumento de la plaza de España en Madrid. Por último, en esta misma sección, tienen su espacio las aportaciones realizadas por el personal científico del CSIC publicadas en editoriales comerciales o en Editorial CSIC. La necesaria selección ha permitido exponer solo una muestra representativa como Usa y Cervantes, El Quijote y el pensamiento teórico-literario, Cervantes genio y libertad, Enciclopedia cervantina, Cervantes, Constantinopla y la Gran Sultana, Cervantes monumento nacional o el ejemplar publicado este mismo año 2016 Una ingeniosa locura. Libros y erudición en Cervantes ${ }^{12}$.

La exposición Cervantes y el CSIC se presentó en el acto en honor de Cervantes realizado el 19 de abril de 2016 y programado en el marco de las actividades con motivo del cuarto centenario de su muerte. El Presidente del CSIC, don Emilio Lora-Tamayo pronunció las palabras de presentación y don Alfredo Alvar la conferencia sobre Cervantes. La biblioteca Tomás Navarro Tomás, representada por su directora, presentó el audiovisual sobre la importancia de Cervantes en el CSIC realizando después una visita guiada a la exposición. El acto finalizó con una lectura dramatizada de Palabra de perro, obra de Juan Mayorga inspirada en El coloquio de los perros de Cervantes, muestra igualmente de la presencia de Cervantes en la literatura de nuestro siglo XXI.

El mismo discurso expositivo se puede encontrar en el portal digital Cervantes y el CSIC que ofrece una visita virtual a la exposición al que se han añadido otros elementos de interés para los estudiosos de Cervantes como el vaciado completo de artículos de la revista Anales Cervantinos, la bibliografía de textos publicados por investigadores del CSIC sobre la vida y obra de Cervantes o el enlace al audiovisual y también al catálogo CIRBIC, desde donde se puede acceder a toda la colección cervantina del CSIC. También en el portal

11. Cervantes Saavedra (1631 y 1738).

12. Dopico y Layna Rans (2009); Garrido Gallardo y Alburquerque (2008); Alvar Ezquerra (2004); Alvar Ezquerra (2005-2016); García Lorenzo (1993); Álvarez Barrientos (2009) disponible en versión electrónica gratuita en http://libros.csic.es/product_info.php?products_id=200 [Consultado el 21-06-2016]; Alvar Ezquerra (2016). 
se ponen a disposición de los usuarios los folletos explicativos, el programa de mano, el tríptico de las actividades y el marcapáginas ${ }^{13}$ diseñado como regalo para los asistentes.

Por último y dentro de las celebraciones en honor de Cervantes organizadas por el CSIC, conviene mencionar también la exposición en paneles explicativos que, con el nombre Conoce a Cervantes a través del CSIC, se ha instalado en el Real Jardín Botánico durante los meses de abril y mayo de 2016. Esta exposición ofrece en dieciocho carteles una adaptación de la exposición Cervantes y el CSIC cuyos ejemplares físicos han sido sustituidos por reproducciones fotográficas de portadas e ilustraciones siguiendo el mismo discurso expositivo. Las dos versiones de la exposición tienen previsto un recorrido por las delegaciones que el CSIC tiene en diferentes comunidades autónomas y por algunos institutos que han mostrado un interés especial en ello.

El conjunto de actividades reunidas bajo el título Cervantes y el CSIC es el homenaje que rinde el Consejo Superior de Investigaciones Científicas al príncipe de las letras en el IV centenario de su muerte gracias a la realización de la Biblioteca Tomás Navarro Tomás en colaboración con la Red de Bibliotecas y Archivos del CSIC y con la Unidad de Recursos de Información para la Investigación Científica, y contando con el apoyo de científicos de diferentes institutos y del personal de las unidades técnicas del Centro de Ciencias Humanas y Sociales y de la Vicepresidencia Adjunta de Cultura Científica del Consejo Superior de Investigaciones Científicas.

\section{BIBLIOGRAFÍA CITADA}

Alvar Ezquerra, Alfredo (2004). Cervantes genio y libertad. Madrid: Temas de Hoy.

Alvar Ezquerra, Alfredo y Sevilla, Florencio (dir.) (2005-2016). Gran Enciclopedia Cervantina. Madrid: Castalia Ediciones.

Alvar Ezquerra, Alfredo (2016). Una ingeniosa locura. Libros y erudición en Cervantes. Madrid: CSIC.

Álvarez Barrientos, Joaquín (2009). Cervantes monumento nacional, Madrid: CSIC, disponible en versión electrónica gratuita en http://libros.csic.es/product_info.php?products_ id $=200$ [Consultado el 21-06-2016]

Benavente, Jacinto (1908). El nuevo coloquio de los perros. Madrid: El Cuento Semanal. Cervantes Saavedra, Miguel de (1631). Novelas exemplares. Barcelona: Esteban Liveròs.

Cervantes Saavedra, Miguel de (1738). Vida y hechos del ingeniosos hidalgo don Quixote de la Mancha, Londres: J. y R. Tonson.

De Haedo, Diego O.S.B. (1612). Topografia e historia general de Argel, Valladolid: por Diego Fernández de Cordoua y Ouiedo, Impressor de libros.

Dopico, Georgina y Layna Rans, Francisco (ed.) (2009). USA Cervantes. 39 cervantistas en Estados Unidos. Madrid: CSIC.

13. El portal digital puede consultarse en http://biblioteca.cchs.csic.es/dia_libro_cervantes_2016/ [Consultado el 21-06-2016] 
Garrido Gallardo, Miguel Ángel y Alburquerque, Luis (2008). El Quijote y el pensamiento teórico-literario. Madrid: CSIC, 2008.

Esquirol, E. (1847). Tratado completo de las enagenaciones mentales. Madrid: Imprenta del Colegio de Sordomudos.

Fernández de Lizardi, José Joaquín (1942). La educación de las mujeres o La quijotita y su prima, México: Cámara Mexicana del Libro.

Fernández de Oviedo, Gonzalo (1851-1855). Historia general y natural de las Indias islas y tierra firme del mar Océano, Madrid, Real Academia de la Historia.

Fischer, Jan J. [1958]. Lisák Pedro Napsal. Praga: [Statní Narkladatelstvi Krasné Literatury, Hudby a Umeni].

García Lorenzo, Luciano (1993), “Cervantes, Constantinopla y la Gran Sultana”, Anales Cervantinos. 31, pp. 201-213.

Gatell, Pedro (1794). Historia del más famoso escudero Sancho Panza. Madrid: Imprenta Real.

Huarte, Juan (1883). Examen de ingenios para las ciencias. Barcelona: Imprenta de la viuda de Subirana.

Laugier de Tassy, Mr. [1750]. Historia del reyno de Argel, Madrid: En la oficina de Pantaleón Aznar.

Peset, José Luis (2010). Las melancolías de Sancho. Madrid: Asociación Española de Neuropsiquiatría.

Peset, José Luis (2015). Melancolía e ilustración: diálogos cervantinos en torno a Cadalso. Madrid: Abada.

Pi y Molist, Emilio (1886). Los primores de don Quijote. Barcelona: Imprenta Barcelonesa.

Ramón y Cajal, Santiago (1905). Psicología de Don Quijote y el quijotismo. Madrid: Imprenta y librería de Nicolás Moya.

Solís, Antonio de (1780). La gitanilla en Madrid. Valencia: Imprenta de Joseph y Thomàs de Orga.

Valverde de Hamusco, Juan (1556). Historia de la composición del cuerpo humano, Roma, Imprenta de Antonio Salamanca y Antonio Lafrerij. 